\title{
Cobalt Nanocluster-Filled Carbon Nanotube Arrays: Engineered Photonic Bandgap and Optical Reflectivity
}

\author{
Caterina Soldano, ${ }^{\dagger}$ Francesco Rossella, ${ }^{\ddagger}$ Vittorio Bellani, ${ }^{\ddagger, *}$ Silvia Giudicatti, ${ }^{\ddagger}$ and Swastik Kar ${ }^{\S}$ \\ ${ }^{\dagger}$ CEMES, Centre d'Elaboration de Matériaux et d’Etudes Structurales, CNRS UPR 801129 rue Jeanne Marvig, Toulouse, France, „Dipartimento di Fisica "A. Volta” and \\ CNISM, Universitá di Pavia, via Bassi 6, 27100 Pavia, Italy, and §Department of Physics, Northeastern University, Boston, Massachusetts 02115, United States
}

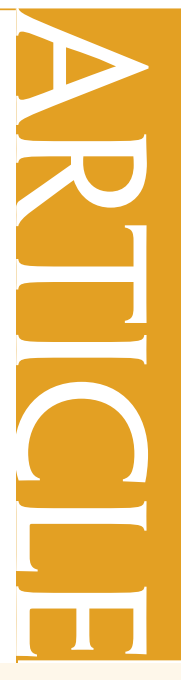

$\mathrm{O}$ ver the past decade or so, the interest of the scientific community in carbon nanotubes (CNTs) has greatly increased because of their potential as novel materials for a variety of applications ranging from nanoelectronic devices to sensors ${ }^{1-3}$ as well as for the study of their fundamental properties. ${ }^{4-6}$

In this context, one area of research that has been gaining growing importance is the design, development, and characterization of carbon nanotube-based hybrid structures, for example, those comprising nanoscale metal particles and carbon nanotubes. Owing to their quantum confined geometry and enhanced surface-to-volume ratio, metallic nanostructures can develop unique electronic, optical, magnetic, and catalytic properties. $^{7-9}$

The combination of metals and their carbides or oxides with carbon nanotubes may give rise to significantly novel electronic, optical, and mechanical properties. ${ }^{10-12}$ Partially or fully filled carbon nanotubes with magnetic materials present both small size and enhanced magnetic coercitivity, ${ }^{13}$ a condition which opens up the way to applications in magnetic data storage technology. In those hybrid materials, the carbon shells provide a valuable barrier against oxidation, ensuring a long-term stability of the ferromagnetic core.

Further, carbon nanotubes also represent an interesting platform to investigate phenomenon like the Kondo effect ${ }^{14-16}$ in the case of magnetic impurities, as well as enhanced spin-orbit coupling. ${ }^{17,18}$ Recently it has been shown that in the case of cobaltnanoclusters-filled MWNTs the charge transport properties of the hosting tube are extremely sensitive to the magnetic state of the Co clusters inside the tube itwww.acsnano.org
ABSTRACT Perfect vertically aligned and periodically arranged arrays of multidielectric heterostructures are ideal platforms both for photonic crystals and photonic bandgap materials. Carbon nanotubes grown inside anodic alumina templates form a novel class of heterostructured materials ideally suited for building such platforms. By engineering metallic (cobalt) nanoclusters inside the nanotubes, we present a novel method for tailoring the photonic bandgap as well as the magnitude of the reflectivity in these systems. We present spectroscopic ellipsometry (SE) and reflectivity measurements to investigate the effect of the presence of cobalt clusters on the optical response of multiwall carbon nanotubes (MWNT) grown in anodized alumina template. The real $\left(\varepsilon_{1}\right)$ and imaginary $\left(\varepsilon_{2}\right)$ part of the pseudodielectric function of the MWNT and Co-MWNT system have been studied in a wide energy range $(1.4-5 \mathrm{eV})$. We found that the cobalt filling modifies the electronic structure of the nanotubes, suggesting that the insertion of the clusters leads to a semiconductor behavior. Angle-resolved reflectivity measurements further show that the metal filling drastically enhances the optical response up to 2 orders of magnitude.

KEYWORDS: carbon nanotube arrays - cobalt clusters-filled tube · spectroscopic enhancers - low reflectivity materials

self. ${ }^{19}$ All these properties render these hybrid materials promising candidates for device applications with multiple and diverse functionalities.

The electronic band structure of a variety of such systems is commonly studied using optical reflectivity techniques, which often require complex and lengthy simulations and numerical analysis to retrieve optical constants..$^{20-22}$ In particular, spectroscopic ellipsometry (SE) is an optical technique that measures the complex ratio $\tilde{\sigma}=\chi_{i} / \chi_{r}\left(=\tan (\Psi) e^{i \Delta}\right)$ between the amplitudes of the polarization states of the reflected and the incident waves, $r$ and $i$, respectively. ${ }^{23}$ Here, $\tan (\Psi)$ represents the amplitude ratio upon reflection, and $\Delta$ is the phase shift. This technique is frequently used for in situ real-time monitoring of molecular beam epitaxy (MBE) growth of semiconductor thin films, heterostructures, and low-dimensional systems. ${ }^{24}$ In the case of
*Address correspondence to
bellani@unipv.it.

Received for review July 28, 2010 and accepted October 01, 2010.

Published online October 11, 2010. $10.1021 / \mathrm{nn} 101801 \mathrm{y}$

() 2010 American Chemical Society

$$
\text { VOL. } 4 \text { - NO. } 11 \text { - 6573-6578 - } 2010 \mathrm{ACSNANO}
$$






Figure 1. (a) Scanning electron microscopy (SEM) image of large diameter tubes grown in alumina template (AAO), where the tubes are only partially released from the template itself. (b) SEM image of MWNTs filled with cobalt clusters (bright areas).

ambient-bulk material interfaces (two-phase model) the ratio $\tilde{\sigma}=\chi_{i} / \chi_{r}$ is directly related to the dielectric function of the material. In nanostructured materials, which are often more complex than multilayered structures, this model no longer entirely applies. Nevertheless, it is very useful to represent the complex reflectance ratio as a pseudodielectric function in a twophase model description. In the case of template-grown tubes, the two-phase model gives an average response of the template-nanotubes system. This methodology has been fruitfully used to study the dielectric and optical properties of nanostructure materials like semiconductor superlattices. ${ }^{25,26}$ Despite its broad applicability, there have been only a very limited number of studies of carbon nanotubes using SE; Fagan and co-workers used transmission ellipsometry to study the polarizability of excitons in this system. ${ }^{27}$ In a previous work, we have explored the electronic transport properties of individual hybrid structures of template-grown multiwall carbon nanotubes (MWNTs). ${ }^{19,28}$ In this work, we present a detailed investigation of both spectroscopic ellipsometry and optical reflectivity of Co-nanoclustersfilled template grown MWNTs. We find that the insertion of the cobalt clusters in the nanotubes leads to (a) a large band gap semiconductor behavior, (b) a great enhancement of the reflectivity signal, and (c) an oscillatory and anisotropic reflectivity which undergoes a significant transformation in both its magnitude and photon-energy dependence.

Multiwall carbon nanotubes (MWNTs) are grown in anodized alumina (AAO) template (diameter $\sim 250 \mathrm{~nm}$ ) by chemical vapor deposition with acetylene precursor gas at a temperature of $650{ }^{\circ} \mathrm{C}$. Tubes grown in template are uniform in size (depending on the template pore diameter) and all vertically aligned in hexagonal array. Compared to other types of nanotubes growth, the template growth does not need any external metal catalyst since the template itself acts as a catalyst.

Figure 1 shows a high magnification scanning electron microscopy (SEM) image of MWNTs grown inside the AAO template, where the template is partially etched to provide better visibility of the nanotubes, while the rest of the template continues to keep the tubes well aligned and isolated from each other. Electrochemistry is then used to insert cobalt clusters inside the nanotube, after the MWNT growth (we refer to those as Co-MWNTs). ${ }^{29,30}$ Compared to electroless ${ }^{31}$ and sol-gel ${ }^{32}$ methods, electrodeposition in nanopore templates allows for a selective deposition of material inside each individual tube. Figure $1 \mathrm{~b}$ is an SEM image of individual Co-MWNTs. Here, the structure has been released completely from the host AAO matrix for imaging purposes, and the bright areas represent metal clusters located within the inner volume of the MWNTs. Note that the SE measurements, both in bare- and Co-filled nanotubes have been performed with the tubes in their perfectly aligned geometry within the template.

\section{RESULTS AND DISCUSSION}

Spectroscopic Ellipsometry. Figure 2 shows the variation of the real $\left(\varepsilon_{1}\right)$ and imaginary part $\left(\varepsilon_{2}\right)$ of the dielectric functions with photon energy ( $1 \mathrm{eV}<E<$ $5 \mathrm{eV}$ ) ranging over the $\mathrm{UV}-$ visible - NIR regions of optical wavelengths. The dielectric functions have been numerically obtained using a two-phase (air/nanostructure) model ${ }^{23,24}$ from the measured SE functions $\tan (\Psi)$ and $\cos (\Delta)$, for both the cobaltfilled and unfilled nanotubes. The complex pseudodielectric function $\varepsilon_{2}$ of the MWNT sample increases gradually with increasing energies and presents a wide peak-like structure $E_{0}$ in the nearultraviolet region at $4.2 \mathrm{eV}$; the real part of the pseudodielectric function $\varepsilon_{1}$ presents, at the same energy, a point of inflection which evidences the dispersive-like line shape of this transition in $\varepsilon_{1}$, as observed in other nanostructured materials. ${ }^{27}$ The energy of this transition suggests the insulating behavior of the MWNT sample, with an optical energy gap that can be estimated, from the rapid increases of $\varepsilon_{2}$, at around $3.2 \mathrm{eV}$. The optical energy gap is estimated from $\varepsilon_{2}$, separating (i) the low energy contribution due to impurities which is responsible of the small linear increase below $3 \mathrm{eV}$, (ii) the sudden ex- 
ponential increase of $\varepsilon_{2}$ related to the onset of the interband fundamental transition, and (iii) the Gaussian $E_{0}$ peaked-structure associated to the interband transition between states of the fundamental energy band at higher energy than the gap. We verified that regimes ii and iii are well fitted by an exponential function and Gaussian line-shapes, respectively; further, at around $3.2 \mathrm{eV}$ a crossover between regimes $\mathrm{ii}$ and iii is found which identifies the optical energy gap.

From the independent electronic transport measurement as a function of temperature in individual AAO-MWNTs, we find that the approximate electronic bandgap is only about $0.055 \mathrm{eV} .^{28}$ This suggests that the photonic transition observed in our samples, whose excitation energy is orders-of-magnitude larger, is not related to the excitations across the narrow electronic bandgap of the individual tubes, but is possibly an outcome of photonic bandgaps of the composite periodic array of MWNTs within the host AAO, which have different effective dielectric constants. The inclusion of cobalt clusters markedly changes the dielectric response, evidencing the modification of the energy band structure of the material. Indeed, in the Co-MWNT the fundamental interband transition $E_{0}$ lies at much lower energy than in the MWNT, and the optical gap, obtained from the rapid increase of $\varepsilon_{2}$ in the left side of the $E_{0}$ structure, is around $2.6 \mathrm{eV}$, red-shifted into the blue region of the spectrum. Thus, the SE data suggest, in the case of the Co-MWNT sample, a wide-gap semiconducting behavior. At higher energy we observe for $\varepsilon_{2}$ the development of a second feature labeled $E_{1}$. The high-energy limit of the experimental setup at $5 \mathrm{eV}$ prevents us from observing the complete evolution of the feature itself. The presence of $E_{1}$ interband transition at higher energy could be either due to a red-shifted structure in the host MWNT array that was beyond $5 \mathrm{eV}$ or an additional structure due to the inclusion of Co clusters that enriches the overall photonic band structure.

Reflectivity. The reflectivity $R$ has been first simulated by finite difference time domain (FDTD) analysis at normal incidence (Figure 3 ) for the unfilled case, and then measured at different incident angles $\left(10^{\circ}<\theta<80^{\circ}\right)$ on the same sample in the Co-filled $\left(R_{\text {Co-MWNT }}\right)$ and unfilled ( $\left.R_{\mathrm{MWNT}}\right)$ areas (Figure 4$)$. In our simulation (see Materials and Method for more details), the sample under investigation is approximated to an ordered array of vertically aligned CNTs with a diameter of $\sim 250 \mathrm{~nm}$, separated one from the other by ca. $20-30 \mathrm{~nm}$, as schematically represented in Figure 3a. Two main behaviors clearly emerge in the calculated reflectance spectrum in Figure $3 b$. First, the very low reflectivity values in the unfilled system $(R<0.01)$, and second, the presence of seemingly periodic oscillations, which are observable in the entire energy range of the simulation (intentionally wider than the one used in the experi-

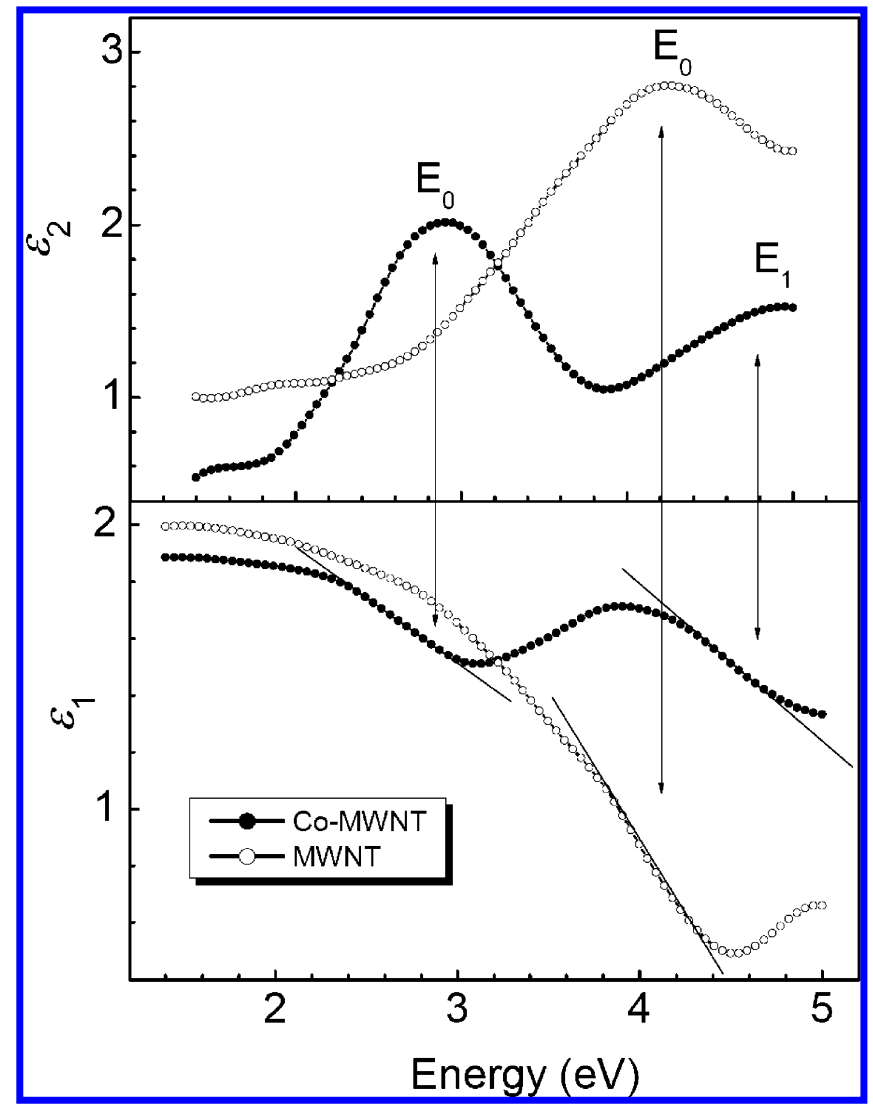

Figure 2. (Top) Imaginary $\varepsilon_{2}$ and (bottom) real $\varepsilon_{1}$ part of the pseudodielectric function for MWNT and Co-MWNT from spectroscopic ellipsometry measurements. The segments in the lower panel show the points of inflection, related to the interband transitions $E_{0}$ and $E_{1}$.

ments) and which have a growing amplitude as energy increases. We have also included some of our experimental results which will be discussed in more details later on, to clearly show the oscillatory motion. Presented data are for incident angle of $10^{\circ}$, which represents the experimental conditions closest to the simulation configuration and are decoupled from the linear trend.

The optical response of carbon nanotubes arrays depends on the atomic structure of each individual tube as well as their overall arrangement. In particular, singlewall carbon nanotubes with a diameter less than $1 \mathrm{~nm}$ present a strong dependence of the chirality of the tube itself. ${ }^{33}$ On the other hand, multiwall carbon nanotubes show a more uniform and regular response, mainly due to their large size. ${ }^{34}$

Further, the optical properties of an array of tubes strongly depend on their arrangement, size, and intertube separation. In fact, in the case of more disordered tubes orientation, the co- and cross-polarized absorbance (parallel and perpendicular to the tube axis, respectively) are extremely sensitive to the alignment of the tubes within the matrix array. ${ }^{35}$ Then, when considering the quality of the MWNT, the presence of disorder in the tube can affect the value of the permittivity 


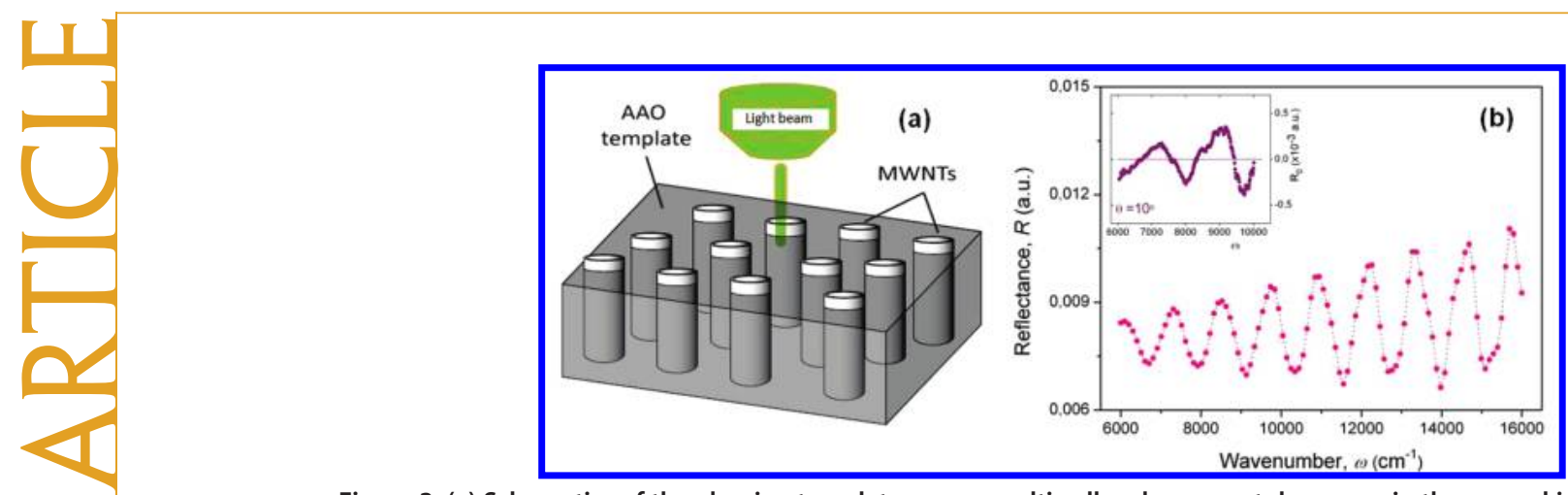

Figure 3. (a) Schematics of the alumina template-grown multiwall carbon nanotubes array in the normal incidence configuration $\left(\theta=0^{\circ}\right)$ for reflectivity measurements. (b) Simulated reflectance spectrum from an ordered array as shown in part a. (Inset) Experimental reflectivity measurement $\left(\theta=10^{\circ}\right)$ clearly shows the oscillatory behavior component.

of the sample, ${ }^{36}$ because those defects behave as polarized centers. It has been shown that arrays of vertically aligned carbon nanotubes behave as an array of charged harmonic oscillators upon light irradiation. ${ }^{24,37}$ Such systems are commonly described by a DrudeLorentz multioscillators system, for which it is possible to write the complex frequency dependent permittivity as a combination of a frequency-independent contribution and a series of charge harmonic oscillators. Further, CVD-grown MWNTs on a Fe-coated silicon substrate present angular-dependent oscillations with increasing degree of (vertical) alignment in the $s$-component of the polarization. ${ }^{22}$

Figure 4 panels $\mathrm{a}$ and $\mathrm{b}$ present the angular dependence of the measured reflectivity in MWNTs and Cofilled MWNTs, respectively. We distinguish two separate effects: (i) the angular dependence and (ii) the effect of the presence of the clusters. In our unfilled sample (MWNT) we identify a general oscillatory behavior, as indicated by the arrows, where the amplitude of those oscillations is quite small and tends to smoothen out with increasing incident angle, while the overall reflec-

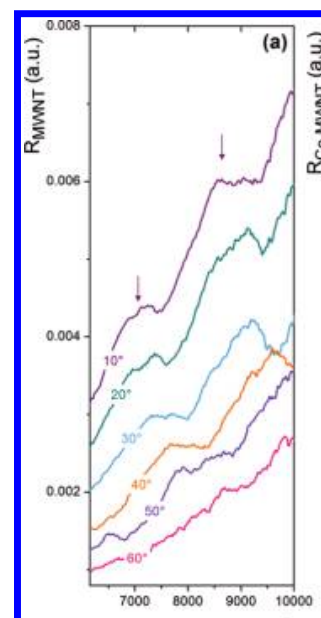

Wavenumbers, $\omega\left(\mathrm{cm}^{-1}\right)$

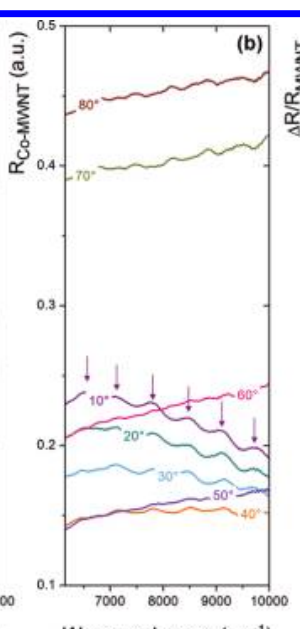

Wavenumbers, $\omega\left(\mathrm{cm}^{-1}\right)$

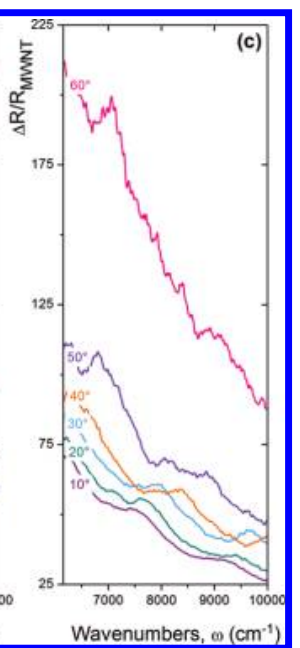

Wavenumbers, $\omega\left(\mathrm{cm}^{-1}\right)$
Figure 4. Angular dependence of the reflectivity of (a) MWNTs, (b) Co-MWNTs, and (c) normalized reflectivity $\left(=\Delta R / R_{\mathrm{MWNT}}\right)$ in the $\left[6000 \mathrm{~cm}^{-1} ; 10000 \mathrm{~cm}^{-1}\right]$. Arrows in panels $a$ and $b$ indicate the observed oscillations. Angles and colors are labeled consistently in the three panels. tivity decreases for increasing angles (Figure 4a). Simple geometrical considerations explain this result: for increasing incident angle, the illuminated area increases, leading to an increased number of "charged" tubes. Thus, the observed smooth and small oscillations are the signature of the alignment of the tubes; in addition, compared to available literature, our sample presents a perfect vertical alignment due to the physical constraint provided by the template itself. It is thus possible to suggest that those oscillations additionally represent individual tubes which are illuminated from the incident light. We can hence assume that the number of oscillations is the number of excited tubes. Thus, if 4-5 tubes ( oscillations) are excited, we can roughly estimate a beam diameter of $1 \mu \mathrm{m}$, a value which is in good agreement with the experimental spot size.

Further, we found that close to normal incidence (i.e., at $10^{\circ}$ ), the reflectivity is well below 0.004 at the lowest photon energy. This result is very consistent with the one reported by Yang et al., ${ }^{38}$ which showed that low-density vertically aligned multiwall carbon nanotubes can have total reflectance as small as $0.045 \%$ due to their low density and high surface roughness. In our case, the nanotubes are much better aligned and the overall surface is much smoother, which may be the reason for the slightly higher reflectivity. Nevertheless, such high absorption of light over a large range of photon energies in the IR range renders those hybrid structures suitable for a number of applications ranging from solar energy harvesting to stealth technologies. ${ }^{39}$ In addition, these structures are much more robust as they are impregnated inside the AAO matrix and hence are far more suitable for long-term applications in extreme conditions.

However, the larger oscillations (indicated by the arrows) observed in the case of Co-MWNTs (Figure 4b) cannot be only considered as the signature of the degree of alignment of the system, but necessarily the result of the effect of the presence of the clusters. In fact, in metal $(\mathrm{Ag})$ partially filled tubes the reflectivity presents collective response and enhancement also identified as surface plasmons..$^{40}$ Further, the reflectivity of our filled tubes could exhibit similar features with re- 
spect to the reflectivity of an array of metal nanoparticles. ${ }^{41}$ The angular dependence of $R_{\text {Co-MWNT }}$ mimics the overall behavior of $R_{\mathrm{MWNT}}$ up to $40^{\circ}$ (signal decreasing for increasing incidence angle); for angles greater than $40^{\circ}$ the dependence of $R_{\text {Co-MWNT }}$ clearly changes, with the onset of an enhancement effect as a function of both increasing wavenumber and increasing angles. We calculated the normalized reflectivity $\left(\Delta R / R_{\mathrm{MWNT}}=\right.$ ( $\left.R_{\text {Co-MWNT }}-R_{\mathrm{MWNT}}\right) / R_{\mathrm{MWNT}}$ as a tool to quantify the effect of the presence of the clusters inside the tubes (Figure 4c). For each incident angle we found that the reflectivity in the case of Co-MWNT is enhanced by factors ranging from $\sim 25$ to $\sim 225$ compared to the MWNT counterpart; this strong enhancement due to the presence of cobalt increases with increasing incident angle and at a fixed angle decreases for increasing energy. We believe that the component of the electric field of the radiation parallel to the tube is the one mostly responsible for inducing a collective response of the Co clusters.

Detailed analysis of the dependence of those oscillations shows that each peak is shifted toward higher wavenumber for increasing incident angles, as shown in Figure 5, where $\omega_{j}$ represents the peak frequency of the jth oscillation. For each peak, we observed a slight increase of the central position $\left(\theta<50^{\circ}\right)$, followed by a smooth decrease. In the case of an error of 5\% (ca. $30-50 \mathrm{~cm}^{-1}$ ) in the determination of the peak position, which nevertheless represents an overestimate, the trend remains unchanged. We note that for the first oscillation, although visible, it is hard to precisely determine the position of the peak itself. This could be the result of the change in the geometry of the illuminated surface when the angle of incidence of the light is changing.

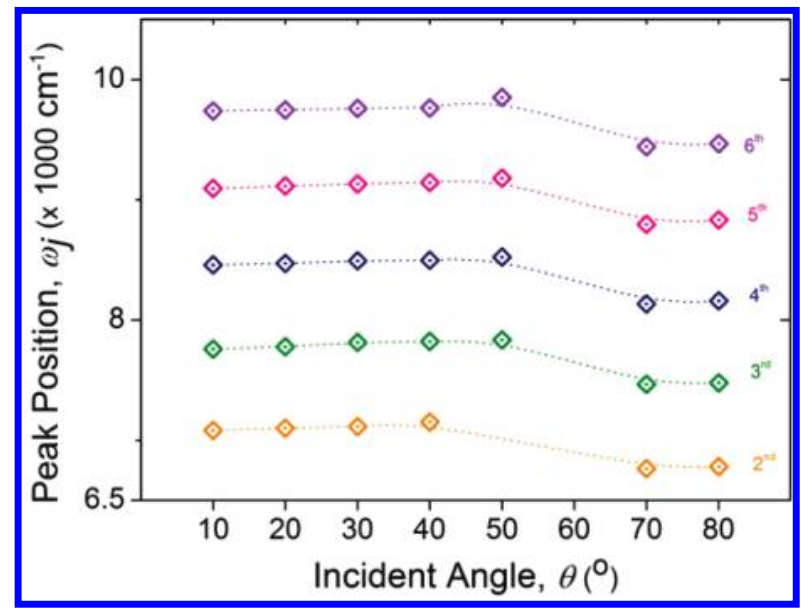

Figure 5. Angular dependence of the position, $\omega_{j}$ of the jth peak $(2<j<6)$, as labeled on the right side, for Co-MWNTs array. Dashed lines represent a guide to the eye.

\section{CONCLUSION}

We have studied the effect of the presence of cobalt clusters on the optical response of multiwall carbon nanotubes grown in alumina template. Spectroscopic ellipsometry measurements reveal that the incorporation of cobalt drastically changes the dielectric response of the material, showing an enrichment of the energy band structure, with evidence of a widegap semiconducting behavior. Measurements of optical reflectivity show an enhancement due to the metal filling and an oscillatory response due to the degree of alignment of the tube, in good qualitative agreement with the simulation. This investigation opens up the way to exploring hybrid AAO-grown MWNT arrays as platforms to develop photonic band gap materials based on enhanced optical response, for which appropriate pore size and/or filling materials could be tailored for selective applications.

\section{MATERIALS AND METHOD}

Spectroscopic ellipsometry spectra have been recorded at room temperature with an automatic rotating polarizer SOPRA MOSS-ES4G instrument, in the spectral range between 1.4 and $5.0 \mathrm{eV}$, with an incidence angle of $75^{\circ}$ using a $\mu$-spot setup which allowed the use of a light spot with a diameter of $1 \mathrm{~mm}$. Variableangle specular reflectance was measured in the $\left[6000 \mathrm{~cm}^{-1}\right.$; $10000 \mathrm{~cm}^{-1}$ ] spectral range by means of a Fourier transform spectrophotometer Bruker IFS66. The light of a Xe arc-lamp was collimated and then focused on the sample into a spot of approximately $1 \mu \mathrm{m}$ in diameter. An in-house built $\theta-2 \theta$ goniometer allows varying the value of the incidence angle between 0 and $80^{\circ}$, with a resolution of $1^{\circ}$.

Finite difference time domain (FDTD) numerical simulations were also performed to calculate the reflectance spectrum of the investigated unfilled sample. It was modeled as a $40 \mu \mathrm{m}$ thick $\mathrm{Al}_{2} \mathrm{O}_{3}$ film with cylindrical holes of radius $125 \mathrm{~nm}$ arranged in a hexagonal lattice of period $280 \mathrm{~nm}$. The carbon nanotubes inside the holes were mimicked by circular rings with inner and outer radii of 115 and $125 \mathrm{~nm}$, respectively, while their height was equal to the entire $\mathrm{Al}_{2} \mathrm{O}_{3}$ membrane thickness. The refractive index of $\mathrm{Al}_{2} \mathrm{O}_{3}$ and carbon nanotubes was set as 1.6 and 1.36, respectively. The carbon nanotubes refractive index was assumed to be isotropic. The investigated system was normally il- luminated by a plane wave source polarized along the $x$-direction and propagating along the $z$-direction. The source spectrum covered the wavelength range $0.625-1.66667 \mu \mathrm{m}$ $\left(6000-16000 \mathrm{~cm}^{-1}\right)$. The reflectance spectrum was recorded by a 2D z-normal frequency domain power monitor characterized by 100 frequency points in the same spectral range of the applied source.

Acknowledgment. This work has been financially supported by the project QUANTDEV of the Foundation Cariplo. C.S. acknowledges the support of a postdoctoral fellowship from the ERC-2007-StG-203872 COMOSYEL project led by E. Dujardin. S.K. acknowledges partial support by NSF ECCS 0925708.

\section{REFERENCES AND NOTES}

1. Avouris, P.; Chen, Z.; Perebeinos, V. Carbon-Based Electronics. Nat. Nanotechnol. 2007, 2, 605-615.

2. Chiu, H.-Y.; Hung, P.; Postma, H. W. C; Bockrath, M. AtomicScale Mass Sensing Using Carbon Nanotube Resonators. Nano Lett. 2008, 8, 4342-4346.

3. Lidorikis, E.; Ferrari., C. Photonics with Multiwall Carbon Nanotube Arrays. ACS Nano 2009, 3, 1238-1248.

4. Saito, R.; Dresselhaus, G.; Dresselhaus, M. S. Physical 
Properties of Carbon Nanotubes; Imperial College Press: London, 1998.

5. Charlier, J.-C.; Blasé, X.; Roche, S. Electronic and Transport Properties of Nanotubes. Rev. Mod. Phys. 2007, 79, 677-732.

6. Semicond. Sci. Technol. 2006, 21 (11),special issue on charge transport in carbon nanotubes.

7. Gräber, M. R.; Weiss, M.; Oberholzer, S.; Schoenenberger, C. Defining and Controlling Double Quantum Dots in Single-Walled Carbon Nanotubes. Semicond. Sci. Technol. 2006, 21, S64-S68.

8. Ravindran, S.; Chaudhary, S.; Colburn, B.; Ozkan, M.; Ozkan, C. S. Covalent Coupling of Quantum Dots to Multiwalled Carbon Nanotubes for Electronic Device Applications. Nano Lett. 2003, 3, 447-453.

9. Pal, S.; Chandra, S.; Phan, M.-H.; Mukherjee, P.; Srikanth, H. Carbon Nanostraws: Nanotubes Filled with Superparamagnetic Nanoparticles. Nanotechnology 2009, 20, 485604.

10. Baughman, R. H.; Zakhidov, A. A.; de Heer, W. A. Carbon Nanotubes-The Route Toward Applications. Science 2002, 297, 787-792.

11. Meyer, R. R.; Sloan, J.; Dunin-Borkowski, R. E.; Novotny, M. C.; Bailey, S. R.; Hutchison, J. L.; Green, M. L. H. Discrete Atom Imaging of One-Dimensional Crystals Formed within Single-Walled Carbon Nanotubes. Science 2000, 289, 1324-1326.

12. Li, D.-C.; Dai, L.; Huang, S.; Mau, A. W. H.; Wang, Z. L. Structure and Growth of Aligned Carbon Nanotube Films by Pyrolysis. Chem. Phys. Lett. 2000, 316, 349-355.

13. Leonhardt, A.; Ritschel, M.; Kozhuharova, R.; Graff, A.; Mühl, T.; Hunle, R.; Mönch, I.; Elefant, D.; Schneider, C. M. Synthesis and Properties of Filled Carbon Nanotubes. Diamond Relat. Mater. 2003, 12, 790-793.

14. Kondo, J. Resistance Minimum in Dilute Magnetic Alloys. Prog. Theor. Phys. 1964, 32, 37-49.

15. Kondo; J. Solid State Physics; Academic Press: New York, 1969; Vol. 23.

16. Guéron, S.; Deshmukh Mandar, M.; Myers, E. B.; Ralph, D. C. Tunneling via Individual Electronic States in Ferromagnetic Nanoparticles. Phys. Rev. Lett. 1999, 83, 4148-4151.

17. Yang, C-K.; Zhao, J.; Lu, J. P. Magnetism of TransitionMetal/Carbon-Nanotube Hybrid Structures. Phys. Rev. Lett. 2003, 90, 257203.

18. Wang, J.; Jo, C.; Wu, R. Magnetic Properties of Fe-5d (Os, Ir, and Pt) Nanowires Encapsulated in Carbon Nanotubes. Appl. Phys. Lett. 2008, 92, 032507.

19. Soldano, C.; Kar, S.; Talapatra, S.; Nayak, S.; Ajayan, P. M. Detection of Nanoscale Magnetic Activity Using a Single Carbon Nanotube. Nano Lett. 2008, 8, 4498-4505.

20. Kamarás, K.; Rinzler, A. G.; Tanner, D. B.; Walters, D. A. Polarization-Dependent Optical Reflectivity in Magnetically Oriented Carbon Nanotube Networks. Phys. Stat. Sol. B 2006, 243, 3126-3129.

21. Dragoman, D.; Dragoman, M. Electromagnetic Wave Propagation in Dense Carbon Nanotube Arrays. J. Appl. Phys. 2006, 99, 076106.

22. de los Arcos, T.; Oelhafen, P; Mathys, D. Optical Characterization of Alignment and Effective Refractive Index in Carbon Nanotube Films. Nanotechnology 2007, 18, 265706.

23. Bashara, M. Ellipsometry and Polarized Light; Elsevier: Amsterdam, 2003.

24. Fujiwara, K. Spectroscopic Ellipsometry: Principles and Applications; Wiley; Amsterdam, 2007.

25. Bellani, V.; Parravicini, G. B.; Diez, E.; Domínguez-Adame, F. Hey, R. Ellipsometric Characterization of Random and Random-Dimer GaAs- $\mathrm{Al}_{x} \mathrm{Ga}_{1-x}$ As Superlattices. Phys. Rev. B 2002, 66, 193310

26. Bellani, V.; Stella, A.; Chen, C.; Wang, X. Spectroscopic Ellipsometry Study of $\mathrm{Cd}_{1-x} \mathrm{Mn}_{x} \mathrm{Te} / \mathrm{CdTe}$ Superlattices. J. Appl. Phys. 2005, 98, 103523.

27. Fagan, J. A.; Simpson, J. R.; Landi, B. J.; Richter, L. J.; Mandelbaum, I.; Bajpai, V.; Ho, D. L.; Raffaelle, R.; Hight
Walker, A. R.; Bauer, B. J.; et al. Dielectric Response of Aligned Semiconducting Single-Wall Nanotubes. Phys. Rev. Lett. 2007, 98, 147402.

28. Kar, S.; Soldano, C.; Chen, Li; Talapatra, S.; Vajtai, R.; Nayak, S.; Ajayan, P. M. Lüttinger Liquid to Al'tshuler-Aronov Transition in Disordered, Many-Channel Carbon Nanotubes. ACS Nano 2009, 3, 207-212.

29. Davis, D.; Podlaha, E. J. CoNiCu and Cu Nanotubes Electrodeposition. Electrochem. Sol. State Lett. 2005, 8, D1D4.

30. Martin, C. R.; Nishizawa, M.; Jirage, K.; Kang, M. Investigations of the Transport Properties of Gold Nanotubule Membranes. J. Phys. Chem. B 2001, 1105, 1925-1934.

31. Martin, C. R. Nanomaterials: A Membrane-Based Synthetic Approach. Science 1994, 266, 1961-1966.

32. Miller, S. A.; Young, V. Y.; Martin, C. R. Electro-osmotic Flow in Template-Prepared Carbon Nanotube Membranes. J. Am. Chem. Soc. 2001, 123, 12335-12342.

33. Bachilo, S. M.; Strano, M. S.; Kittrell, C; Hauge, R. H.; Smalley, R. E.; Weisman, R. B. Structure-Assigned Optical Spectra of Single-Walled Carbon Nanotubes. Science 2002, 298, 2361-2366.

34. Lin, M. F. Optical Spectra of Single-Wall Carbon Nanotube Bundles. Phys. Rev. B 2000, 62, 13153-13159.

35. Ni, C; Bandaru, P. R. Enhanced Optical Absorption CrossSection Characteristics of Multiwall Carbon Nanotubes. Carbon 2009, 47, 2898-2903.

36. Watts, P. C. P.; Hsu, W.-K.; Barnes, A.; Chambers, B. High Permittivity from Defective Multiwalled Carbon Nanotubes in the X-Band. Adv. Mater. 2003, 15, 600-603.

37. Kamaras, K.; Pekker, A.; Bruckner, M.; Borondics, F.; Rinzler, A. G.; Tanner, D. B; Itkis, M. E.; Haddon, R. C.; Tan, Y.; Resasco, D. E. Wide-Range Optical Spectra of Carbon Nanotubes: A Comparative Study. Phys. Stat. Sol. B 2008, 245, 2229-2232.

38. Yang, Zu-Po; Ci, Lijie; Bur, J. A.; Lin, S. Y.; Ajayan, P. M. Experimental Observation of an Extremely Dark Material Made by a Low-Density Nanotube Array. Nano Lett. 2008, 8, 446-451.

39. Ong, Pang-Leen; Euler, W. B.; Levitsky, I. A. Hybrid Solar Cells Based on Single-Walled Carbon Nanotubes/Si Heterojunctions. Nanotechnology 2010, 21, 105203.

40. García-Vidal, F. J; Pitarke, J. M.; Pendry, J. B. Silver-Filled Carbon Nanotubes Used as Spectroscopic Enhancers. Phys. Rev. B 1998, 58, 6783-6786.

41. Mertens, H.; Verhoeven, J.; Polman, A.; Tichelaar, F. D. Infrared Surface Plasmons in Two-Dimensional Silver Nanoparticle Arrays in Silicon. Appl. Phys. Lett. 2004, 85 1317-1319. 